\title{
Endocan (ESM-1) levels in gingival crevicular fluid correlate with ICAM-1 and LFA- 1 in periodontitis
}

\author{
Mahmure Ayşe TAYMAN ${ }^{(a)}$ \\ Canan ÖNDER(b) \\ Şivge KURGAN ${ }^{(b)}$ \\ Muhittin Abdulkadir SERDAR(c) \\ Meral GÜNHAN(b) \\ (a)Ankara Yıldırım Beyazıt University, Faculty \\ of Dentistry, Department of Periodontology, \\ Ankara, Turkey. \\ (b)Ankara Uiversity, Faculty of Dentistry, \\ Department of Periodontology, \\ Ankara, Turkey. \\ (c)Acıbadem University, School of Medicine, \\ Department of Medical Biochemistry, \\ Ankara, Turkey.
}

Declaration of Interests: The authors certify that they have no commercial or associative interest that represents a conflict of interest in connection with the manuscript.

Corresponding Author:

Mahmure Ayşe Tayman

E-mail: ayseatay06@hotmail.com

hitps://doi.org/10.1590/1807-3107bor-2021.vol35.0005

Submitted: July 1, 2019

Accepted for publication: July 27, 2020

Last revision: August 18, 2020

\begin{abstract}
Endocan, a $50 \mathrm{kDa}$ soluble proteoglycan, also called endothelial cell-specific molecule-1 (ESM-1), is involved in many major cellular activities and has been reported to be overexpressed in inflammatory conditions. This study aims to determine ESM-1 levels in gingival crevicular fluid (GCF) samples from individuals with periodontitis to determine the correlation between the levels of lymphocyte-function-associated antigen-1 (LFA-1), intercellular adhesion molecule-1 (ICAM-1), and clinical findings of periodontitis. This study enrolled 27 individuals diagnosed with Stage III-Grade C generalized periodontitis and 16 individuals as healthy controls. Bleeding on probing (BOP), probing pocket depth (PPD), and clinical attachment loss (CAL) were calculated. Enzyme-linked immunosorbent assay (ELISA) test was used for detecting the levels of ESM-1, ICAM-1, and LFA-1 in GCF samples. PPD, BOP, CAL, and GCF volumes were significantly increased in patients with periodontitis in comparison to the control group ( $p<0.001$ ). The total amount of ESM-1, ICAM-1, and LFA-1 levels in GCF were increased in the periodontitis group $(\mathrm{p}<0.001)$. ESM-1 level correlated with PPD, BOP, and CAL ( $\mathrm{p}<0.05)$. ICAM-1 level correlated with BOP and CAL $(p<0.05)$. LFA-1 level correlated with PPD and CAL $(p<0.05)$. Our data indicate that ESM-1, ICAM-1, and LFA-1 levels are increased in GCF of patients with periodontitis. These molecules could be associated with the pathogenesis and progression of periodontal disease.
\end{abstract}

Keywords: Intercellular Adhesion Molecule-1; Lymphocyte Function-Associated Antigen-1.

\section{Introduction}

Periodontitis is an inflammatory disease that can be modified by microbiological, immunological, genetic, and environmental risk factors. ${ }^{1}$ Inflammation occurs as a host response to pathogens and includes events such as vascular dilation, papillary permeability, and extravasation of leukocytes. The first cells to reach the inflammation site are polymorphonuclear leukocytes (PMNLs). Endothelial cells are responsible for the migration of leukocytes against microbial biofilm. ${ }^{2}$ During the first stage of host defense, ${ }^{3}$ PMNLs are involved in the immune response to periodontopathogens in the gingival sulcus. If there are 
abnormalities in the number and/or functions of PMNLs, rapid and severe periodontal destruction may occur, and in this case, the presence of the protective PMNL barrier becomes important. ${ }^{4}$

Endothelial adhesion of circulating leukocytes and migration to inflammatory sites is a prominent step in the onset and progression of periodontal lesions. ${ }^{5}$ The endothelium has important functions in inflammation, angiogenesis, coagulation, and tumor invasion, mainly via the regulation of receptor/ligand interactions and release of several mediators. Cell adhesion molecules (CAMs) are cell-surface proteins associated with cell binding. Attaching leukocytes to endothelial cells via CAMs requires inflammationmediated signaling pathways. ${ }^{6,7} \mathrm{CAM}$ levels are also positively related to the degree of inflammation in periodontal tissues. ${ }^{6}$ Proinflammatory cytokines, which are produced and released in response to infection, lead to the production of vascular cell adhesion molecule-1 (VCAM-1) and intercellular adhesion molecule-1 (ICAM-1) throughout the endothelium. ${ }^{8}$ ICAM-1 and VCAM-1 belong to the immunoglobulin superfamily, and their expression is provided by leukocytes, endothelial cells, monocytes, synovial cells, fibroblasts, and epithelial cells. They have an effect on cell adhesion, leukocyte collection, immunological events, and inflammatory reactions, resulting in rounding and transmigration of leukocytes. ${ }^{9}$

Lymphocyte function-associated antigen-1 (LFA-1), one of the ligands on the surface of leukocytes, enables their binding to CAMs in the endothelium. ${ }^{10}$ LFA-1 is one of the adhesion molecules present on lymphocytes and other leukocytes, belongs to the integrin superfamily, and acts as a key factor in the migration of leukocytes into tissues. The binding of LFA-1 to ICAM-1 is an important step for the tight adhesion and transmigration of leukocytes and also stimulates signaling pathways important for T-cell differentiation. ${ }^{11}$

Endothelial cell-specific molecule-1 (ESM-1) is one of the molecules secreted by endothelial cells. ${ }^{12}$ ESM-1 is a $50 \mathrm{kDa}$ soluble proteoglycan involved in many major cellular activities, such as cell transformation, cell proliferation, VCAM- 1 and ICAM-1 expression, regulation of leukocyte migration, neovascularization, and metastasis of tumors. ${ }^{12,13,14}$ ESM-1 has been reported to be overexpressed in inflammatory conditions, cardiovascular diseases, sepsis, cancer, and obesity, ${ }^{15}$ and has been shown to increase the secretion of VCAM-1, E-selectin, and ICAM-1. Therefore, ESM-1 has been designated as a proinflammatory mediator that binds to LFA-1 on human leukocytes and regulates LFA-1 interactions with ICAM-1. ${ }^{12,14,16,17}$

Previous evidence indicates that ESM-1 is increased in inflammatory diseases, ${ }^{12,13,15,18}$ and inflammation has already been shown to have an important contributing part in the pathogenetic changes in periodontitis. The hypothesis is that ESM-1 may have an effect on periodontal inflammation and may have a role in the pathogenesis of periodontitis. Türer et al. ${ }^{19}$ evaluated the role of ESM-1 in periodontal inflammation. They studied its association with tumor necrosis factor alpha and vascular endothelial growth factor and found that these markers were increased in periodontal inflammation. However, CAMs have an important function in transmigration and are reported to be regulated by ESM-1. To date, there is no known study examining the association of ESM-1 levels with CAMs in GCF in periodontitis. Thus, this study aims to measure ESM-1 levels in GCF samples from individuals with Stage III-Grade $C$ generalized periodontitis and determine correlations among the levels of ICAM-1 and LFA-1 and clinical signs of periodontitis.

\section{Methodology}

\section{Patient selection}

The present study was carried out between June 2016 and January 2018 in the Ankara University Faculty of Dentistry, Department of Periodontology, Ankara, Turkey. Individuals who applied to the periodontology outpatient clinic for dental treatment or gingival examination were allocated to the study. This study enrolled 27 systemically healthy patients having Stage III-Grade $C$ periodontitis and 16 periodontally and systemically healthy individuals (control group). The 1975 Declaration of Helsinki was followed. Ethical approval of the study (dated June 28, 2016, and numbered 36290600/60) was obtained from the 
Ankara University Faculty of Dentistry Clinical Research Ethics Committee. The aim of the study and the study procedure were explained to each participant. At the beginning of the study, each patient signed an informed consent form.

\section{Exclusion and inclusion criteria}

Patients with systemic diseases (such as diabetes mellitus, rheumatoid arthritis and obesity), pregnant women, lactating mothers, and smokers were excluded from the study. In addition, patients who had taken any antibiotics or anti-inflammatory drugs during the past six months or who were subjected to any periodontal treatment were also excluded. Inclusion criteria were determined by radiographic examination and full-mouth clinical periodontal evaluation. All involved individuals were systemically healthy. Individuals without allergic, inflammatory, or autoimmune disease were also included in the study.

\section{Clinical measurements}

Clinical attachment loss (CAL), probing pocket depth (PPD), and bleeding on probing (BOP) of every tooth obtained from the six areas (mesiobuccal, midbuccal, distobuccal, mesiolingual, mid-lingual, and distolingual) were determined by a single experienced periodontologist (M.A.T.) using a periodontal sond (Williams' probe, Hu-Friedy, Chicago, IL, USA). Prior to the study, the examiners were calibrated. A total of five volunteers were assessed twice, leaving a one-hour time period between assessments. Between the recordings, the second set was performed by blinding out the initial one, and the reproducibility assessment resulted in $85 \%$ of sites for which repeated probing meant that measurements were within $\pm 1 \mathrm{~mm}$. A reference examiner (M.G.), who has more than 20 years of experience in periodontology, has calibrated our periodontist (M.A.T.). Scores of probing depth demonstrated good reproducibility as assessed by an inter-examiner $(\mathrm{K}=0.826)$. PPD measurement was registered to the nearest millimeter, and each survey close to $0.5 \mathrm{~mm}$ was rounded down to the next lower number. CAL was calculated with the PPD and recession values. The average score for whole-mouth PI, GI, PPD, CAL, and the count of sections with BOP divided by the total count of sections per mouth and centuplicated were determined for every patient. Each patient had at least 20 teeth other than the third molar tooth.

Clinical diagnosis was considered according to the "2017 World Workshop on the Classification of Periodontal and Peri-Implant Diseases and Conditions." ${ }^{20}$ Periodontal health (control group) was defined as a PPD of $\leq 3 \mathrm{~mm}$ and BOP $(+)$ of $\leq 10 \%$. Patients who had interdental radiographic bone loss of $\geq 2 \mathrm{~mm}$ on nonadjacent teeth or buccal or oral radiographic bone loss up to $15 \%$ with probing depth of $>3 \mathrm{~mm}$ for $\geq 2$ teeth were diagnosed as having periodontitis. The periodontitis group consisted of patients with Stage III-Grade C generalized periodontitis.

\section{Measurement of gingival crevicular fluid}

Gingival crevicular fluid (GCF) samples were collected from three single rooted teeth of each eligible subject. Specimens were collected from mesial and distal regions with PPD $>5 \mathrm{~mm}$ for periodontitis groups. Teeth with single root were picked randomly in the control group. First, the region was isolated to avoid saliva contamination. With the aid of a sterile curette, the supragingival plaque was cautiously removed and the tooth surfaces were slowly dried with air. Paper strips (Periopaper, Oraflow Inc., Plainview, USA) were used to collect GCF samples, placed in the crevice until mild resistance was sensed, and held in that position for 30 seconds. Strips stained with blood were excluded. To sample without mechanical stimulation of the tissues, paper strips were cautiously immersed in the sulcus/pocket orifice (extrasulcular technique). ${ }^{21}$ The amount of GCF was measured using a calibrated device (Periotron 8000, Oraflow Inc., New York, USA). Strips were placed in previously encoded polypropylene microcentrifuge tubes. Numerical values read by the device were converted to volume (microliter) according to a standard curve. The electronic gingival fluid measuring device findings were transformed into a genuine volume (microliter) relative to the standard curve. The whole GCF samples were preserved at $-80^{\circ} \mathrm{C}$ until analyzed by enzyme-linked immunosorbent assay (ELISA). 


\section{GCF ELISA method for ESM-1, LFA-1, and ICAM-1}

ESM-1, LFA-1, and ICAM-1 levels in GCF samples were analyzed by human ELISA kits. All assay procedures were performed based on the guidelines of the producer. GCF samples were decompounded from the strips with the aid of a centrifugal procedure. Separation was applied by adding $200 \mu 1$ of sample (test) buffer found in the kit materials. Afterward, the strips and the buffer inside the microcentrifuge tubes were centrifuged for 20 minutes at $3000 \times \mathrm{g}$. Following centrifugation, the strips were detached and the residual fluid in the tubes was analyzed for ESM-1, LFA-1, and ICAM-1 using the abovementioned commercial ELISA kits. Crevicular ESM-1, LFA-1, and ICAM-1 levels in every specimen were determined using the concentration data of standards in the kit materials. The acquired concentration value for every specimen was fixed for the genuine volume of GCF by dividing the volume of ESM-1, LFA-1, and ICAM- 1 by the sample volume; the results were stated as ng of LFA-1, $\mu$ g of ICAM-1, and $\mu$ g of ESM-1 per site. The intra- and interassay precision values for LFA- 1 were $<10 \%$ and $<12 \%$, respectively; the corresponding values for ICAM- 1 were $<10 \%$ and $<12 \%$ and that for ESM- 1 were $<10 \%$ and $<12 \%$. Limit of detection was $0.268 \mathrm{ng} / \mathrm{ml}$ for LFA-1, 20.11 $\mu \mathrm{g} / \mathrm{L}$ for ICAM-1, and 7.506 $\mu \mathrm{g} / \mathrm{L}$ for ESM-1.

\section{Statistical analysis}

We used PASW (version 18) to analyze the data. We pooled 15 patients from each group on the basis of a power calculation of $80 \%$ power to define the least clinically significant difference $(0.4 \mathrm{~mm})$ in probing depth, with $5 \%$ type I error. The original numbers were 27 for the Stage III-Grade $\mathrm{C}$ generalized periodontitis group and 16 for the control group. Shapiro-Wilk test was implemented to determine data normality. The Student's $t$-test was used for comparison of periodontitis and control groups. Correlations between clinical periodontal parameters and GCF ESM-1, ICAM-1, and LFA-1 levels were assessed with Pearson correlation coefficient. The age of the participants was analyzed by Pearson chi-squared test. $p<0.05$ was the level of statistical significance.

\section{Results}

\section{Clinical parameters}

There was no statistically significant variation between the groups in terms of mean age $(p=0.327)$ and in relation to sex distribution between the groups $(\mathrm{p}=0.432)$. In this study, 27 systemically healthy individuals in the Stage III-Grade C periodontitis group (11 males, 16 females; mean \pm standard deviation $[\mathrm{SD}]=38.41 \pm 5.91$ years) and 16 healthy individuals (control group) (8 males, 8 females; mean $\pm \mathrm{SD}=36.20 \pm 8.46$ years) without findings of systemic diseases or periodontitis were enrolled. PPD, BOP, CAL, and GCF values were significantly elevated for the periodontitis group compared to the control group $(p<0.001)$. Table 1 presents the clinical findings.

Table 1. Comparison of clinical periodontal parameters and GCF volumes between periodontitis and healthy groups.

\begin{tabular}{lccc}
\hline \multirow{2}{*}{ Clinical parameters } & Stage III Grade C generalized periodontitis & Control & $p$-value \\
\cline { 2 - 3 } Age (year) & $(\mathrm{CP} ; \mathrm{n}=18)$ & $(\mathrm{C} ; \mathrm{n}=16)$ & 0.327 \\
Gender (female/male) & $38.41 \pm 5.91$ & $36.20 \pm 8.46$ & 0.432 \\
PPD (mm) & $16 / \mathrm{nov}$ & $8 / 8$ & $\mathrm{p}<0.001$ \\
BOP (\%) & $3.76 \pm 0.61^{*}$ & $1.39 \pm 0.20$ & $\mathrm{p}<0.001$ \\
CAL (mm) & $69.99 \pm 16.96^{*}$ & $8.72 \pm 4.25$ & $\mathrm{p}<0.001$ \\
GCF $(\mu \mathrm{l})$ & $4.23 \pm 0.79^{*}$ & $1.46 \pm 0.25$ & $\mathrm{p}<0.001$ \\
\hline
\end{tabular}

PPD: probing pocket depth; BOP: bleeding on probing; CAL: clinical attachment loss; GCF: gingival crevicular fluid. Data expressed as mean \pm SD. Statistically significant $p<0.05$. 


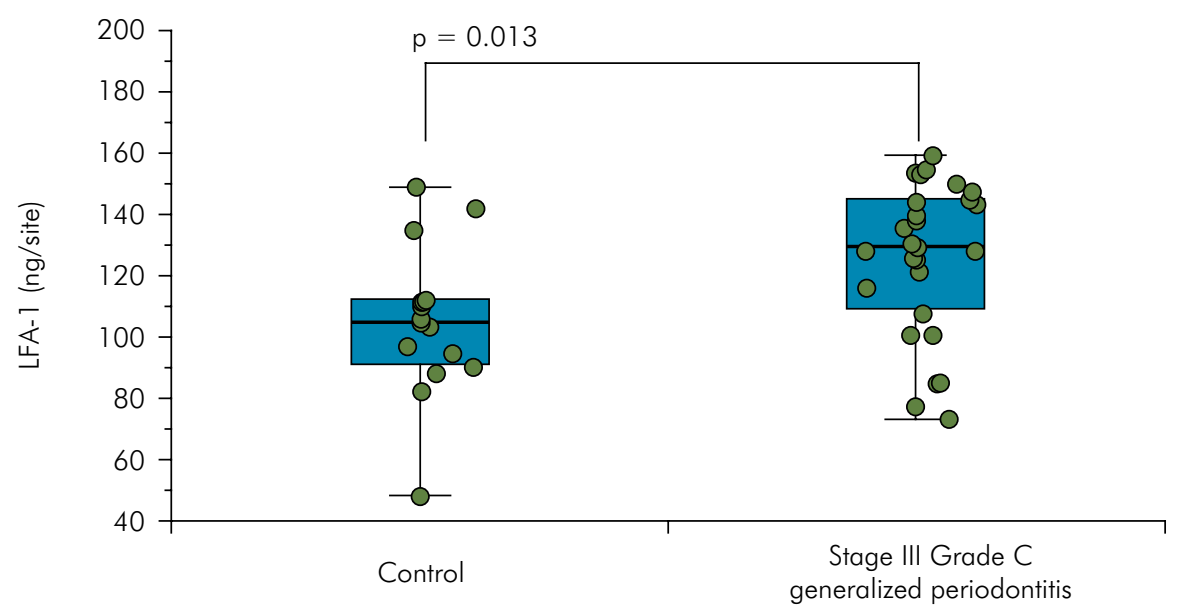

Figure 1. GCF levels of LFA-1 for periodontitis and control groups.

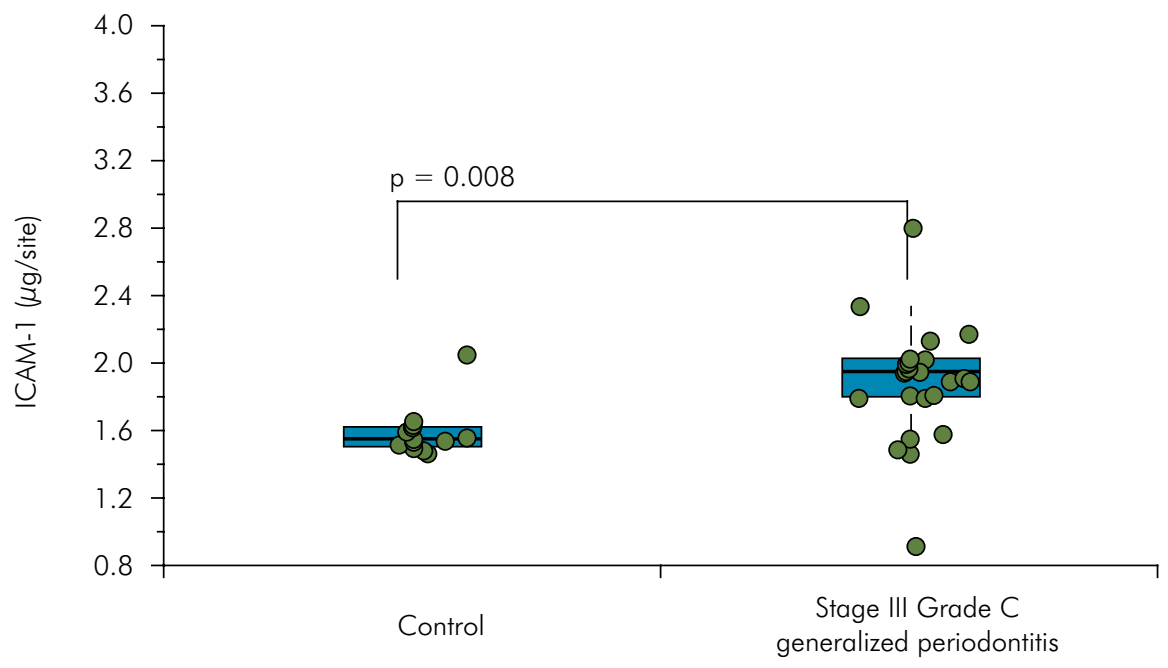

Figure 2. GCF levels of ICAM-1 for periodontitis and control groups.

\section{Biochemical findings}

The total amount of LFA-1, ICAM-1, and ESM-1 were significantly increased in the periodontitis group $(\mathrm{p}<0.001)$ in comparison to the control group (Figures 1, 2, and 3, respectively).

\section{Correlations}

Table 2 shows correlation coefficients between the periodontal clinical parameters and GCF contents of ESM-1, ICAM-1, and LFA-1. A significant relationship was determined between ESM-1 level and PPD, BOP, and CAL ( $p<0.05)$; between ICAM-1 level and BOP and CAL $(p<0.05)$; and between LFA-1 level and PPD and CAL $(\mathrm{p}<0.05)$.

\section{Discussion}

In this study, ESM-1, LFA-1, and ICAM-1 levels of GCF samples of periodontitis were evaluated and compared with a control group. This is the first study to ascertain a relationship between ESM-1 and CAM levels in periodontitis. In accordance with the hypothesis of the study, the values of all 


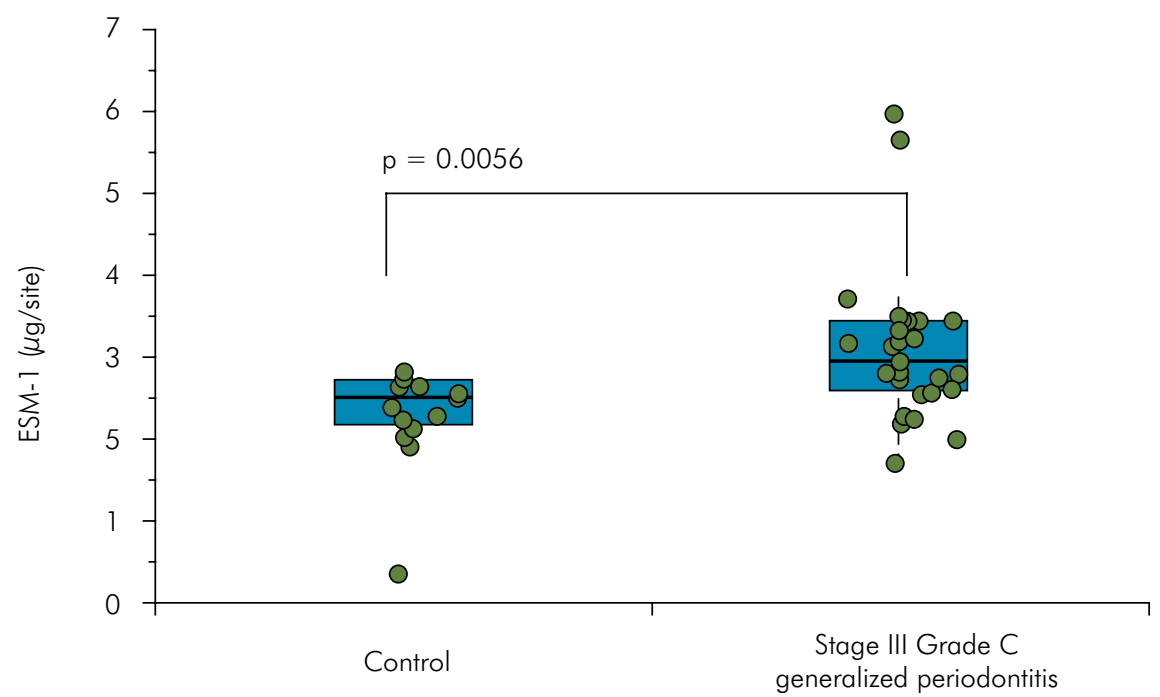

Figure 3. GCF levels of Endocan for periodontitis and control groups.

Table 2. Correlations between GCF ESM-1, ICAM-1, LFA- 1 levels, and periodontal clinical parameters.

\begin{tabular}{|c|c|c|c|}
\hline Parameters & ESM- $1(\mu \mathrm{g} /$ site $)$ & ICAM-1 ( $\mathrm{g} / \mathrm{site})$ & LFA-1 (ng/site) \\
\hline ICAM-1 ( $\mathrm{g} / \mathrm{s} / \mathrm{site})$ & 0.256 & - & - \\
\hline LFA- 1 (ng/site) & 0.198 & $0.416^{*}$ & - \\
\hline PPD (mm) & $0.372^{*}$ & 0.303 & $0.366^{*}$ \\
\hline BOP (\%) & $0.317^{*}$ & $0.336^{*}$ & 0.298 \\
\hline $\mathrm{CAL}(\mathrm{mm})$ & $0.338^{*}$ & $0.359^{*}$ & $0.379^{*}$ \\
\hline Age (year) & -0.053 & $0.330^{*}$ & 0.136 \\
\hline GCF $(\mu l)$ & $0.307^{*}$ & $0.408^{*}$ & $0.338^{*}$ \\
\hline
\end{tabular}

Pearson's correlation, r. *Significant at the 0.05 level.

parameters were increased in the periodontitis group compared to the control group.

Gingival sulcus contains serum-like GCF that includes inflammatory cells and mediators in individuals who have periodontitis versus healthy tissue, and the flow rate and content of GCF are linearly correlated with the severity of inflammation. ${ }^{22}$ Therefore, it is a beneficial diagnostic tool to ascertain the pathological changes of periodontal disease. ${ }^{22} \mathrm{In}$ our study, the volume of GCF was remarkably elevated in the periodontitis group compared to the control group. This result is consistent with the knowledge that the volume of GCF increases in relation to the severity of inflammation. ${ }^{19}$ In assessing the pathogenesis of periodontal diseases, GCF itself and inflammationrelated molecules in GCF are diagnostic and prognostic value. ${ }^{23}$ Studies have shown that GCF biomarkers are more reliable with regard to the total amount of GCF due to the increase of GCF volume and the dilution effect in diseased regions. ${ }^{6,24,25}$ Therefore, in our study, biomarkers were calculated as total amount. Numerous studies have shown high levels of inflammatory mediators and adhesion molecules in areas affected by periodontitis. ${ }^{6,26}$ Consistent with the hypothesis of our study, total amounts of ICAM1, LFA-1, and ESM-1 in GCF were determined to be significantly elevated in individuals with periodontitis compared to the control group.

LFA-1/ICAM-1 binding, which is necessary for extravasation and migration of neutrophils to peripheral tissues, plays an important role in periodontal inflammation. ${ }^{27,28}$ In our study, the high 
level of LFA-1 in GCF of patients with periodontitis, which increased with the severity of inflammation, can be considered as a marker of inflammation. ICAM-1 is an available molecule on leukocytes for intracellular signaling and increases the release of cytokines, ${ }^{29}$ while these cytokines also increase the ICAM-1 level with a double-sided effect. ${ }^{30}$ Hannigan et al. ${ }^{6}$ reported that soluble ICAM- 1 is released as a result of cell damage, and high ICAM-1 levels in the regions affected by periodontitis reflect increased cell damage. Additionally, Pischon et al. ${ }^{11}$ have indicated that CAMs are potential candidate markers for endothelial dysfunction. In another study, the soluble forms of CAMs (sCAMs) level in GCF were evaluated in terms of periodontal health and disease, and as a result, sCAM levels were found to increase with the severity of inflammation. ${ }^{6}$ Our findings were consistent with the results of this study. In accordance with the hypothesis of our study, the ESM-1 levels in GCF was significantly elevated in individuals diagnosed with periodontitis in comparison to the control group. ESM-1 is classified as a proteoglycan produced by vascular endothelial cells and can be considered as an indicator of endothelial activation. ${ }^{14}$ Lee et al ${ }^{16}$ have shown that ESM-1 leads to proinflammatory responses such as hyperpermeability, CAM expression upregulation, and leukocyte adhesion. Lipopolysaccharides are effective in the release of ESM- $1,{ }^{16}$ and ESM- 1 has been reported to stimulate the expression of VCAM-1, ICAM-1, and E-selectin by inducing endothelial cell activation. ${ }^{18}$ It is suggested that ESM-1 contributes to vascular dysfunction by upregulating inflammatory pathways. ${ }^{14}$ In our study, ESM-1 levels in GCF were determined to be significantly higher in individuals with periodontal disease, supporting the fact that this proteoglycan has a proinflammatory effect. Türer et al. ${ }^{19}$ investigated ESM-1 levels in periodontal disease for the first time and found that ESM-1 levels in GCF and serum were significantly elevated in patients with chronic periodontitis compared to the control group. They concluded that ESM-1 can be a diagnostic and prognostic indicator of periodontal disease and may be an inflammatory marker. ${ }^{19}$

In the present study, a positive and significant correlation was found between GCF ESM-1 levels and clinical periodontal parameters (PPD, CAL, and $\mathrm{BOP})$. These results indicate that all three markers change in relation to the severity of inflammation. On the other hand, there was no correlation between the total amount of ESM-1, ICAM-1, and LFA-1 levels. All three markers had proinflammatory effects, and a correlation between these three markers was expected. In addition to the proinflammatory effect of ESM-1, it is also reported that it binds to LFA-1, disrupts ICAM-1/ LFA-1 interaction, and tends to inhibit transmission of leukocytes to the inflammatory site. ${ }^{13,17,31}$ ESM-1 not only induces the release of CAMs from the endothelium by activating proinflammatory signaling pathways but also inhibits the transmigration of circulating leukocytes by preventing ICAM-1/LFA-1 interaction. Therefore, we could say that ESM-1 might have two different pathways related to the severity of inflammation. However, it is unknown which function of ESM-1 in the GCF content is involved in periodontal inflammation. In fact, the reaction and progression of many parameters in periodontitis is complex. Many mediators acting on the periodontal lesion can have a different contribution through changing their biological potential at different stages. Pawlak et al..$^{32}$ reported that ESM-1 is released by the activated endothelium and may have a protective mechanism that disrupts leukocyte adherence to the endothelium.

Our study is important as it is the first study to investigate the correlation of total amounts of GCF ESM-1, ICAM-1, and LFA-1 in periodontal disease. However, the lack of correlation between these markers raises the question of what function ESM-1 has in periodontal inflammation. Future studies are required to clarify whether leukocyte extravasation-inducing effects or a limiting effect is more prominent in the relationship between ESM-1, sCAM, and endothelial dysfunction in periodontal disease.

\section{Conclusion}

This study was the first to show increased ESM1, sICAM-1, and LFA-1 levels in GCF in patients with periodontitis. ESM-1 is a new class of natural endothelial-derived proteoglycan that can regulate CAM interactions and function in patients with 
periodontitis. However, to fully understand the role of ESM-1 in the pathogenesis of periodontal disease, further studies are required to examine ESM-1 levels at different stages of periodontitis and to investigate the impact of periodontal therapy on ESM-1 levels.

\section{Acknowledgements}

This study was supported by the Ankara University Scientific Research Projects Office, Ankara, Turkey with the project number 17B0234002. The authors are grateful to Ankara University Scientific Research Projects Office for their substantial support.

\section{References}

1. Hasturk H, Kantarci A. Activation and resolution of periodontal inflammation and its systemic impact. Periodontol 2000. 2015 Oct;69(1):25573. https://doi.org/10.1111/prd.12105

2. Muller WA. Leukocyte-endothelial-cell interactions in leukocyte transmigration and the inflammatory response. Trends Immunol. 2003 Jun;24(6):32734. https://doi.org/10.1016/S1471-4906(03)00117-0

3. Del Fabbro M, Francetti L, Pizzoni L, Weinstein RL. [Congenital neutrophil defects and periodontal diseases]. Minerva Stomatol. 2000 Jun;49(6):293311. Italian.

4. Deas DE, Mackey SA, McDonnell HT. Systemic disease and periodontitis: manifestations of neutrophil dysfunction. Periodontol 2000. 2003;32(1):82104. https://doi.org/10.1046/i.0906-6713.2003.03207.x

5. Freire MO, Van Dyke TE. Natural resolution of inflammation. Periodontol 2000. 2013 Oct;63(1):14964. https://doi.org/10.1111/prd.12034

6. Hannigan E, O'Connell DP, Hannigan A, Buckley LA. Soluble cell adhesion molecules in gingival crevicular fluid in periodontal health and disease. J Periodontol. 2004 Apr;75(4):54650. https://doi.org/10.1902/jop.2004.75.4.546

7. Wetering S, Berk N, Buul JD, Mul FP, Lommerse I, Mous R, et al. VCAM-1-mediated Rac signaling controls endothelial cell-cell contacts and leukocyte transmigration. Am J Physiol Cell Physiol. 2003 Aug;285(2):C34352. https://doi.org/10.1152/ajpcell.00048.2003

8. Sprague AH, Khalil RA. Inflammatory cytokines in vascular dysfunction and vascular disease. Biochem Pharmacol. 2009 Sep;78(6):53952. https://doi.org/10.1016/i.bcp.2009.04.029

9. Adams DH, Shaw S. Leucocyte-endothelial interactions and regulation of leucocyte migration [Review]. Lancet. 1994 Apr;343(8901):8316. https://doi.org/10.1016/S0140-6736(94)92029-X

10. Mazerolles F, Lumbroso C, Lecomte $O$, Le Deist F, Fischer A. The role of lymphocyte function-associated antigen 1 (LFA-1) in the adherence of T lymphocytes to B lymphocytes. Eur J Immunol. 1988 Aug;18(8):122934. https://doi.org/10.1002/eji.1830180813

11. Pischon N, Hägewald S, Kunze M, Heng N, Christan C, Kleber BM, et al. Influence of periodontal therapy on the regulation of soluble cell adhesion molecule expression in aggressive periodontitis patients. J Periodontol. 2007 Apr;78(4):68390. https://doi.org/10.1902/jop.2007.060286

12. Lassalle P, Molet S, Janin A, Heyden JV, Tavernier J, Fiers W, et al. ESM-1 is a novel human endothelial cell-specific molecule expressed in lung and regulated by cytokines. J Biol Chem. 1996 Aug;271(34):2045864. https://doi.org/10.1074/ibc.271.34.20458

13. Roudnicky F, Poyet C, Wild P, Krampitz S, Negrini F, Huggenberger R, et al. Endocan is upregulated on tumor vessels in invasive bladder cancer where it mediates VEGF-A-induced angiogenesis. Cancer Res. 2013 Feb;73(3):1097106. https://doi.org/10.1158/0008-5472.CAN-12-1855

14. Sarrazin S, Adam E, Lyon M, Depontieu F, Motte V, Landolfi C, et al. Endocan or endothelial cell specific molecule-1 (ESM-1): a potential novel endothelial cell marker and a new target for cancer therapy. Biochim Biophys Acta. 2006 Jan;1765(1):2537.

15. Yilmaz MI, Siriopol D, Saglam M, Kurt YG, Unal HU, Eyileten T, et al. Plasma endocan levels associate with inflammation, vascular abnormalities, cardiovascular events, and survival in chronic kidney disease. Kidney Int. 2014 Dec;86(6):121320. https://doi.org/10.1038/ki.2014.227

16. Lee W, Ku SK, Kim SW, Bae JS. Endocan elicits severe vascular inflammatory responses in vitro and in vivo. J Cell Physiol. 2014 May;229(5):62030. https://doi.org/10.1002/icp.24485

17. Béchard D, Scherpereel A, Hammad H, Gentina T, Tsicopoulos A, Aumercier M, et al. Human endothelial-cell specific molecule-1 binds directly to the integrin CD1 la/CD18 (LFA-1) and blocks binding to intercellular adhesion molecule-1. J Immunol. 2001 Sep;167(6):3099106. https://doi.org/10.4049/jimmunol.167.6.3099

18. Adekola H, Romero R, Chaemsaithong P, Korzeniewski SJ, Dong Z, Yeo L, et al. Endocan, a putative endothelial cell marker, is elevated in preeclampsia, decreased in acute pyelonephritis, and unchanged in other obstetrical syndromes. J Matern Fetal Neonatal Med. 2015;28(14):162132. https://doi.org/10.3109/14767058.2014.964676 
19. Türer ÇC, Durmuş D, Balli U, Güven B. Effect of non-surgical periodontal treatment on gingival crevicular fluid and serum endocan, vascular endothelial growth factor-a, and tumor necrosis factor-alpha levels. J Periodontol. 2017 May;88(5):493501. https://doi.org/10.1902/jop.2016.160279

20. Tonetti MS, Greenwell H, Kornman KS. Staging and grading of periodontitis: framework and proposal of a new classification and case definition. J Clin Periodontol. 2018 Jun;45 Suppl 20:S14961. https://doi.org/10.1111/icpe.12945

21. Loe H, Holm-Pedersen P. Absence and presence of fluid from normal and inflamed gingivae. Periodontics. 1965 Jul-Aug;3:1717.

22. Silvana P. Barros, Ray Williams, Steven Offenbacher, Thiago Morelli. Gingival crevicular as a source of biomarkers for periodontitis. Periodontol 2000. 2016 Feb;70(1):5364. https://doi.org/10.1111/prd.12107

23. Buduneli N, Kinane DF. Host-derived diagnostic markers related to soft tissue destruction and bone degradation in periodontitis. J Clin Periodontol. 2011 Mar;38 Suppl 11:85105. https://doi.org/10.1111/j.1600-051X.2010.01670.x

24. Lin SJ, Chen YL, Kuo MY, Li CL, Lu HK. Measurement of gp130 cytokines oncostatin M and IL-6 in gingival crevicular fluid of patients with chronic periodontitis. Cytokine. 2005 May;30(4):1607. https://doi.org/10.1016/j.cyto.2004.12.018

25. Lamster IB, Oshrain RL, Fiorello LA, Celenti RS, Gordon JM. A comparison of 4 methods of data presentation for lysosomal enzyme activity in gingival crevicular fluid. J Clin Periodontol. 1988 Jul;15(6):34752. https://doi.org/10.1111/j.1600-051X.1988.tb01010.x

26. Oliveira S, Rosowski EE, Huttenlocher A. Neutrophil migration in infection and wound repair: going forward in reverse. Nat Rev Immunol. 2016 May;16(6):37891. https://doi.org/10.1038/nri.2016.49

27. Ley K, Laudanna C, Cybulsky MI, Nourshargh S. Getting to the site of inflammation: the leukocyte adhesion cascade updated. Nat Rev Immunol. 2007 Sep;7(9):67889. https://doi.org/10.1038/nri2156

28. Phillipson M, Kubes P. The neutrophil in vascular inflammation. Nat Med. 2011 Nov;17(11):138190. https://doi.org/10.1038/nm.2514

29. Lukacs NW, Strieter RM, Elner VM, Evanoff HL, Burdick M, Kunkel SL. Intercellular adhesion molecule-1 mediates the expression of monocyte-derived MIP-1 alpha during monocyte-endothelial cell interactions. Blood. 1994 Mar;83(5):11748. https://doi.org/10.1182/blood.V83.5.1174.1174

30. Figenschau SL, Knutsen E, Urbarova I, Fenton C, Elston B, Perander M, et al. ICAMI expression is induced by proinflammatory cytokines and associated with TLS formation in aggressive breast cancer subtypes. Sci Rep. 2018 Aug;8(1):11720. https://doi.org/10.1038/s41598-018-29604-2

31. Kali A, Shetty KS. Endocan: a novel circulating proteoglycan. Indian J Pharmacol. 2014 Nov-Dec;46(6):57983. https://doi.org/10.4103/0253-7613.144891

32. Pawlak K, Mysliwiec M, Pawlak D. Endocan: the new endothelial activation marker independently associated with soluble endothelial adhesion molecules in uraemic patients with cardiovascular disease. Clin Biochem. 2015 Apr;48(6):42530. https://doi.org/10.1016/i.clinbiochem.2015.01.006 